\title{
Dextran-based biodegradable nanoparticles: an alternative and convenient strategy for treatment of traumatic spinal cord injury
}

This article was published in the following Dove Press journal:

International Journal of Nanomedicine

\author{
Wei Liu',* \\ Peng Quan ${ }^{2, *}$ \\ Qingqing $\mathrm{Li}^{1}{ }^{1 *}$ \\ Pengyu Tang' \\ Jian Chen' \\ Tao Jiang ${ }^{3}$ \\ Weihua Cai' \\ 'Department of Orthopaedics, \\ The First Affiliated Hospital \\ of Nanjing Medical University, \\ Nanjing, Jiangsu 210029, China; \\ ${ }^{2}$ Department of Pharmaceutical \\ Science, School of Pharmacy, Shenyang \\ Pharmaceutical University, Shenyang, \\ China; ${ }^{3}$ Department of Orthopaedics, \\ Wuxi People's Hospital Affiliated to \\ Nanjing Medical University, Wuxi, \\ Jiangsu 214023, China \\ *These authors contributed equally \\ to this work
}

\begin{abstract}
Introduction: After traumatic spinal cord injury (SCI), an inhibitory environment that contains chondroitin sulfate proteoglycans (CSPGs) is formed that prevents axonal regeneration and growth.
\end{abstract}

Materials and methods: As previously reported, local administration of $\mathrm{Taxol}^{\circledR}$ at a low concentration has shown promising abilities to promote axonal regeneration and downregulate inhibitory molecules after acute SCI. However, the application of an invasive miniosmotic pump to deliver Taxol and the Cremophor-related toxicity caused by Taxol limits the administration of Taxol.

Results: In this study, the sustained release of paclitaxel (PTX) for 7 days was achieved by incorporating PTX into acetalated dextran (Ac-DEX) nanoparticles, and the prepared PTX-loaded Ac-DEX (PTX@Ac-DEX) nanoparticles promoted neurite extension in the presence of CSPGs. In a rat SCI model, both PTX@Ac-DEX and Taxol enhanced neural regeneration, inhibited CSPGs, protected the injured spinal cord, and improved locomotor recovery. Because of the sustained release of PTX, single administration of PTX@Ac-DEX showed equal therapeutic effect with Taxol, which need to be administered for seven days using a surgically implanted miniosmotic pump.

Conclusion: Overall, this study provides an effective and convenient strategy for SCI therapy, which can improve neurite extension across an inhibitory environment and avoid Cremophorrelated toxicity caused by Taxol.

Keywords: acetalated dextran, nanoparticle, paclitaxel, spinal cord injury

\section{Introduction}

Traumatic spinal cord injury (SCI) is a major medical problem that affects approximately 180,000 patients worldwide each year, ${ }^{1}$ often resulting in motor deficits, bladder dysfunctions, and even death. ${ }^{2}$ SCI is the result of an initial contusion or compression injury followed by a secondary injury that leads to the formation of a glial scar and the upregulation of inhibitory factors, such as chondroitin sulfate proteoglycans (CSPGs) and myelin-associated inhibitors (MAIs), which limit axonal extension and further behavioral recovery. ${ }^{3,4}$ Current studies on SCI mainly focus on rebuilding functional synapses by promoting the intrinsic growth capacity of neurons, as well as downregulating inhibitory factors, removing the extrinsic barriers and minimizing the lost spinal tissues..$^{5-7}$ However, many of these studies only showed limited and unsatisfactory therapeutic efficacy. ${ }^{8}$

Following SCI, microtubule stability is necessary for cell survival, intracellular signaling, and axonal transport. ${ }^{9}$ A study has shown that continuous intrathecal infusion
Correspondence: Weihua Cai Department of Orthopaedics, The First Affiliated Hospital of Nanjing Medical University, 300 Guangzhou Road, Gulou Qu, Nanjing, Jiangsu 210029, China Email caiwhspine@sina.com 
of low-dose Taxol $^{\circledR}$ at the injured site for seven days could cause axonal regeneration and enhance functional recovery in a spinal cord contusion injury model. ${ }^{9}$ Taxol is a clinically approved anticancer drug that inhibits mitosis and stabilizes microtubule formation. Because of the extreme poor solubility of paclitaxel (PTX) in water and many other pharmaceutically acceptable solvents, Taxol is dissolved in Cremophor EL, which can induce peripheral neuropathy. ${ }^{10}$ Therefore, a novel strategy, which can eliminate Cremophor-related toxicity and continuously deliver PTX to the injured site for seven days, will improve the clinical outcome significantly.

Multifunctional therapeutic systems are getting more and more attention in SCI therapy for their unique advantage of combining multistrategy to overcome inhibitory barriers and further enhance functional recovery. ${ }^{11-14}$ Drug delivery systems are engineered technologies, and formulations for the controlled release of therapeutic agents at their target to exert their desired therapeutic effects safely. ${ }^{15}$ An ideal drug delivery system would achieve a sustained release of therapeutics over a long period of time with a favorable release rate. ${ }^{15}$ A variety of drug delivery systems using hydrogels, ${ }^{16,17}$ nanoparticles, ${ }^{18,19}$ and scaffolds ${ }^{12,20}$ as carriers have been proposed by incorporating therapeutic agents, such as monosialoganglioside, methylprednisolone, and neurotrophic factors, to achieve a sustained drug release at the injury site.

In our previous study, ${ }^{16}$ we developed an in situ gelling drug delivery system for the treatment of SCI, which was composed of a Poloxamer-407 and Poloxamer-188 mixturebased thermoresponsive hydrogel matrix and an incorporated therapeutic compound (monosialoganglioside, GM1). A biodegradable water-insoluble polymer, acetalated dextran (Ac-DEX), was recently synthesized by modifying hydroxyl groups of water-soluble dextran with 2-methoxypropene. ${ }^{21,22}$ Ac-DEX and its derivatives have been used in biomedical applications, such as immunotherapy ${ }^{23}$ and myocardial infarction, ${ }^{24}$ for the formulation of drug delivery systems. Our earlier study ${ }^{25}$ demonstrated that Ac-DEX microspheres could protect the injured neurons and as a result promote locomotor recovery by sequestering glutamate and calcium in cerebrospinal fluid (CSF) after traumatic SCI.

In this study, due to the well-established preparation methods and the reported biomedical applications, we wondered if PTX could be incorporated into the neuroprotective Ac-DEX microspheres and single administration of PTX-loaded Ac-DEX (PTX@Ac-DEX) could acquire equal therapeutic effect with Taxol, which was administrated by an invasive intrathecal miniosmotic pump. The aim of this study was to develop a biomaterial-based drug delivery system for the sustained release of PTX by using Ac-DEX nanoparticles to avoid the invasive application of miniosmotic pump and to eliminate Cremophor-related toxicity and explore an effective strategy for SCI therapy.

\section{Materials and methods Preparation of Ac-DEX and PTX@Ac- DEX nanoparticles}

Ac-DEX nanoparticles were prepared using microprecipitation method. Briefly, Ac-DEX (20 mg) was dissolved in ethanol $(1 \mathrm{~mL})$, then the solution was dropwise dripped into the Poloxamer-407 solution ( $5 \mathrm{~mL} ; 1 \%$, w/v) under stirring. The supernatant of the obtained nanosuspension was removed by centrifugation (relative centrifugal force 20,000, 3 minutes) and washed twice with $1 \times$ PBS.

To optimize the PTX@Ac-DEX formulation, Ac-DEX (20 mg) was dissolved in ethanol (1 mL), and PTX (1, 2, or $4 \mathrm{mg}$ ) was added. After that, the ethanol solution containing Ac-DEX and PTX was dropwise dripped into the Poloxamer407 solution $(5 \mathrm{~mL} ; 1 \%$ w/v) under stirring to form the PTX@Ac-DEX nanoparticles.

\section{Characterization of nanoparticles}

The morphology of nanoparticles was evaluated by transmission electron microscope (TEM, Jeol 1400). The TEM samples were prepared by depositing $2 \mu \mathrm{L}$ of the nanoparticle suspensions $(1.0 \mathrm{mg} / \mathrm{mL})$ onto carbon-coated copper grids (300 mesh; Electron Microscopy Sciences, Hatfield, PA, USA). Samples were blotted away after 5 minutes of incubation, and the grids were then washed twice with distilled water and air-dried prior to imaging.

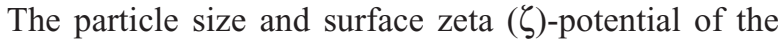
nanoparticles were analyzed using dynamic light scattering with a Zetasizer Nano ZS (Malvern Instruments, Malvern, UK). For measuring the particle size, $1 \mathrm{~mL}$ of the sample was put in a disposable polystyrene cuvette (Sarstedt AG \& Co., Nümbrecht, Germany), and the determination was recorded as the average of three measurements. For measuring the $\zeta$-potential, the samples were measured using disposable folded capillary cells (DTS1070, Malvern Instruments) at $\mathrm{pH} 7.4$ after proper dilution.

The encapsulation efficiency was calculated by normalizing the amount of PTX in the nanoparticles to the amount initially added. The loading fraction of PTX was calculated by measuring the amount of PTX in the nanoparticles and the total weight of the nanoparticles. Briefly, PTX@AcDEX (1 mg) was dissolved in chloroform (1 mL) before acetonitrile/water mixture $(85: 15,9 \mathrm{~mL})$ was added. Afterward, to evaporate the chloroform, a nitrogen stream was added at room temperature. The final solution was processed 
using an absorbance microplate reader at $227 \mathrm{~nm}$ compared to a standard curve. The in vitro release of PTX from PTX@ Ac-DEX was determined by placing PTX@Ac-DEX into the artificial cerebrospinal fluid (aCSF) $(10 \mathrm{~mL})$ to simulate the central nervous system (CNS) environment at $37^{\circ} \mathrm{C}$. Under constant shaking (100 rpm), aCSF of $200 \mu \mathrm{L}$ was withdrawn at specific time points, and the same volume of aCSF was added back to replace the withdrawn volume. The amount of PTX released from PTX@Ac-DEX was determined by high-performance liquid chromatography using Agilent 1,100 (Agilent Technologies, Santa Clara, CA, USA). A C 18 column (Agilent Technologies) was used as the stationary phase and set at $30^{\circ} \mathrm{C}$. The mobile phase was composed of water and acetonitrile $(15: 85, \mathrm{v} / \mathrm{v})$, and the flow rate was $1.0 \mathrm{~mL} / \mathrm{min}$. The injection volume was $50 \mu \mathrm{L}$, and the detection wavelength was $227 \mathrm{~nm}$.

\section{In vitro study}

\section{Culture of neurons and astrocytes}

According to an established protocol, ${ }^{26}$ primary neurons were collected from embryonic (E16-18) Sprague Dawley (SD) rats. In brief, cerebral cortices were isolated and dissociated with trypsin $(0.25 \%$, w/v; Thermo Fisher Scientific, Waltham, MA, USA) for 20 minutes. Neurons were seeded at a density of $1 \times 10^{5} / \mathrm{mL}$ for immunofluorescent staining in 24-well culture plates and $1.5 \times 10^{4} / \mathrm{mL}$ for viability assays on 96-well culture plates. Neurons were maintained in fresh neurobasal medium (Thermo Fisher Scientific) containing 2\% B27 (2\%, w/v; Thermo Fisher Scientific), 1\% glutamine (Thermo Fisher Scientific), $100 \mathrm{IU} / \mathrm{mL}$ penicillin, and $100 \mathrm{mg} / \mathrm{mL}$ streptomycin. Half of the medium was changed every 3 days. After 5 days of cell culture, the obtained neurons were examined by microtubule-associated protein-2 (MAP2; 1:500, mouse IgG1; Abcam, Cambridge, MA, USA) under a fluorescence microscope.

Primary astrocytes were obtained from ScienCell (ScienCell Research Laboratories, Carlsbad, CA, USA). They were harvested into poly-D-lysine-coated culture plates and maintained in astrocyte medium supplemented with $2 \%$ fetal bovine serum, $1 \%$ astrocyte growth supplement (ScienCell Research Laboratories), and 1\% penicillin/streptomycin solution (ScienCell Research Laboratories). Half of the medium was changed every other day. Both neurons and astrocytes were maintained in a standard incubator at $37^{\circ} \mathrm{C}$ with an atmosphere of $5 \%(\mathrm{v} / \mathrm{v}) \mathrm{CO}_{2}$ and $95 \%$ relative humidity.

\section{Cell viability assay}

The viability of neurons and astrocytes was evaluated with a Cell Counting Kit-8 assay (Dojindo, Kumamoto, Japan).
After 12, 24, 48, 72, and 96 hours of incubation, the wells were rinsed three times with $1 \times$ PBS. Then, CCK- 8 solution (10 $\mu \mathrm{L} ; 1: 10$ diluted) in neurobasal medium was added and incubated for 2 hours at $37^{\circ} \mathrm{C}$. The optical absorbance was measured at $450 \mathrm{~nm}$ using an absorbance microplate reader (ELx800, BioTek, Winooski, VT, USA).

\section{In vivo study}

\section{Contusive $\mathrm{SCl}$ model}

All procedures were conducted according to the Guidelines for the Care and Use of Laboratory Animals and were approved by the Animal Care and Use Committee of Nanjing Medical University. Female rats (170-220 g) were anesthetized with chloral hydrate $(350 \mathrm{mg} / \mathrm{kg}$ of body weight). After animals were unresponsive, skin and muscles were opened on the back to expose the vertebral column. A T10 laminectomy was performed, and the exposed dorsal surface of the cord was subjected to weight drop impact using a 10-g rod (2.5 mm in diameter, C4p01-001; RWD Life Science Corp., Shenzhen, China) dropped from a height of $12.5 \mathrm{~mm} \cdot{ }^{27}$ The muscles were sutured immediately after administration, and the skin was then closed. The bladders of animals were manually voided three times per day until the reflexive control of bladder function was restored.

\section{Administration of empty Ac-DEX nanoparticles, Taxol, and PTX@Ac-DEX}

Rats were randomly assigned into the following four groups: SCI, Ac-DEX, Taxol, and PTX@Ac-DEX. With the exception of Taxol, all formulations were intrathecally administered within 5 minutes post-trauma. After loading into a sterilized 26G Hamilton syringe, PBS, Ac-DEX, or PTX@ Ac-DEX were injected approximately $1 \mathrm{~mm}$ rostral and caudal to the lesion epicenter. After each injection, the needle was maintained for an additional 2 minutes to minimize the leakage of injected formulations. Alzet miniosmotic pumps (model \#2004), a rat intrathecal catheter (Alzet \#0007740), and three separate pieces of Silastic tubing (Dow Corning \#508-004) were used for the administration of Taxol. Taxol was administrated at a dose of $256 \mathrm{ng}$ /day for seven days after injury, whereas about $15 \mu \mathrm{g}$ PTX@Ac-DEX diluted in $10 \mu \mathrm{L}$ PBS was administrated after injury.

\section{Assessment of locomotor capacity}

Locomotion recovery after SCI was scored according to the Basso, Beattie, and Bresnahan (BBB) open field 21-point locomotion rating scale. ${ }^{28}$ The movements of hindlimbs were assessed weekly by two independent examiners blinded to the treatment regimen. 


\section{Spinal cord tissue immunofluorescence}

Primary antibodies used in this study included NF200 (1:50, mouse IgG1; Abcam), GFAP (1:1,000, rabbit IgG1; Abcam), and CS56 (1:350, mouse IgM; Abcam). The secondary antibody used was Cy3- or FITC-conjugated secondary antibody (1:200; Jackson ImmunoResearch Laboratories, Inc., West Grove, PA, USA). First, spinal cord sections at day 28 postinjury were permeabilized for 30 minutes in Triton X-100 PBS solution $(0.3 \%, \mathrm{w} / \mathrm{v})$ and then blocked with natural goat serum PBS solution $(10 \%, \mathrm{v} / \mathrm{v})$. The specimens were incubated with primary antibodies overnight at $4^{\circ} \mathrm{C}$, triple washed with PBS, and then incubated with secondary antibody for 2 hours at room temperature. After triple washing with PBS, nuclei were stained with DAPI, and fluorescent images were taken. For each slide, the lesion was identified as the area lacking staining. We selected six different areas near the traumatic lesion as the near-injury area. Six different areas at least 10-mm distance from the traumatic lesion were chosen as far-injury area. The average intensity of NF200, GFAP, and CS56 was measured with ZEN lite software. Data are expressed as the percentage of intensity increase or decrease in the nearinjury area compared with the far-injury area. All images were taken at the same exposure time and conditions.

\section{CSF sampling}

We collected CSF with a 1-mL syringe equipped with a $25 \mathrm{G}$ disposable needle $\left(0.5 \times 20 \mathrm{~mm}^{2}\right)$ as described previously. ${ }^{29}$ First, we washed the rat's head with soap and water, removed the hair with a shaving blade, and fixed the head at an angle of about $135^{\circ}$. Then, the occipital crest was located, and the needle was carefully inserted from the caudal end at $30^{\circ}$ to the body in the muscle gap at $3 \mathrm{~mm}$ below the occipital crest. The tip of the needle was slowly moved to the cerebellum until a depth of about $0.5 \mathrm{~cm}$. When the needle touched the yellow ligament, a sense of resistance was felt followed by a hollow feeling as the needle pierced the yellow ligament. At this time, the tip of the needle reached the cerebellum into the pool. The needle was immediately retracted, and 80-100 $\mu \mathrm{L}$ CSF was slowly extracted in less than 1 minute. The detailed CSF sampling is illustrated in Video S1. The obtained samples were immediately centrifuged to remove any blood and PTX@Ac-DEX nanoparticles that might be present, then stored at $-80^{\circ} \mathrm{C}$ before analysis.

\section{Quantification of PTX in CSF}

The PTX concentration in the CSF was analyzed with liquid chromatography tandem mass spectrometry (LC-MS/MS). The LC system comprised a Shimadzu liquid chromatography system (Shimadzu Co., Kyoto, Japan) equipped with a binary pump (LC-30AD), an autosampler (SIL-30AC), a column oven (CTO-20A), a system controller (CBM-20A), and a degasser (DGU-20A). Mass spectrometric analysis was performed using an AB SCIEX API6500 + triple-quadrupole instrument (Ontario, Canada) with an electron spray ionization interface. Data acquisition and control system were created by using Analyst 1.6.2 software from AB SCIEX (Framingham, MA, USA). Chromatographic separation was performed on a $\mathrm{C} 18$ column (XBridge UPLC BEH C18 $[2.1 \times 50 \mathrm{~mm}, 2.5 \mu \mathrm{m}]$ ), with water as mobile phase A (containing $0.025 \% \mathrm{FA}$ and $1 \mathrm{mM} \mathrm{NH} 4 \mathrm{OAc}$ ) and acetonitrile as mobile phase $\mathrm{B}$ (containing $0.025 \% \mathrm{FA}$ and $1 \mathrm{mM} \mathrm{NH} 4 \mathrm{OAc}$ ). The column was eluted at a flow rate of $0.8 \mathrm{~mL} / \mathrm{min}$ in a gradient program consisting of $10 \%$ phase $\mathrm{B}(0-0.2$ minute), $10 \%$ to $95 \% \mathrm{~B}(0.2-0.6$ minute), $95 \% \mathrm{~B}(0.6-1.20$ minute), $95 \%$ to $10 \% \mathrm{~B}$ (1.20-1.21 minute), and 10\% B (1.21-1.80 minute). The injection volume was $2 \mu \mathrm{L}$. The retention time for PTX and internal standard (Glipizide) were 0.85 minute and 0.80 minute, respectively. The precursor and product ion pairs were $\mathrm{m} / \mathrm{z} 876.4 \rightarrow 591.1$ for PTX and $\mathrm{m} / \mathrm{z} 446.2 \rightarrow 321.1$ for Glipizide. An aliquot of $6 \mu \mathrm{L}$ sample was diluted with $24 \mu \mathrm{L} 80 \%$ MeOHaq. For protein precipitation, $200 \mu \mathrm{L}$ internal standard (Glipizide, $50 \mathrm{ng} / \mathrm{mL}$ in acetonitrile) was added. The mixture was vortexed for 10 minutes at $750 \mathrm{rpm}$ and centrifuged at $6,000 \mathrm{rpm}$ for 10 minutes. An aliquot of $2 \mu \mathrm{L}$ supernatant was injected for LC-MS/MS analysis.

\section{Statistical analysis}

Data were expressed as mean \pm standard deviation for at least three independent experiments. Multiple group comparisons were made using one-way analysis of variance followed by Bonferroni's post hoc test. * represented a $P$-value $<0.05$, ** represented a $P$-value $<0.01$, and $* * *$ represented a $P$-value $<0.001$.

\section{Results \\ Optimization of PTX@Ac-DEX composition}

The typical transmission electron microscopy image of empty Ac-DEX nanoparticles is presented in Figure 1A, showing a spherical morphology. According to the dynamic light scattering result (Figure 1B-E), incorporation of PTX into Ac-DEX nanoparticles did not change the size and $\zeta$-potential of the nanoparticles. All the obtained nanoparticles had an average particle size of approximately $175 \mathrm{~nm}$, with a narrow size distribution (polydispersity index, PDI, is an approximate value) and negatively charged surface (approximately $-10 \mathrm{mV}$ at $\mathrm{pH}$ 7.4). The PDI value was $0.08533 \pm 0.00153$ at $1: 5,0.06533 \pm 0.01518$ at $1: 10$, $0.09867 \pm 0.02892$ at $1: 20$, and $0.069 \pm 0.02987$ for empty 
A

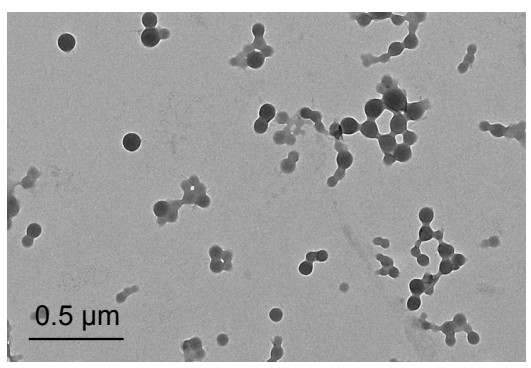

B

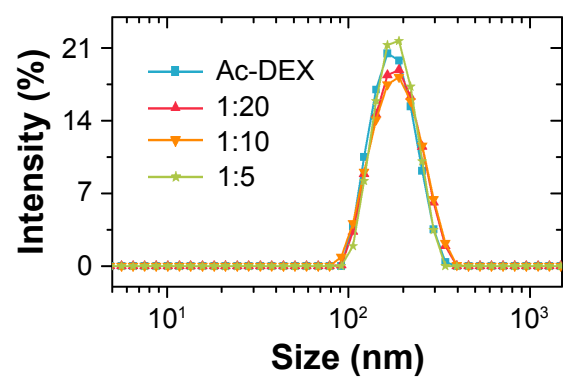

C

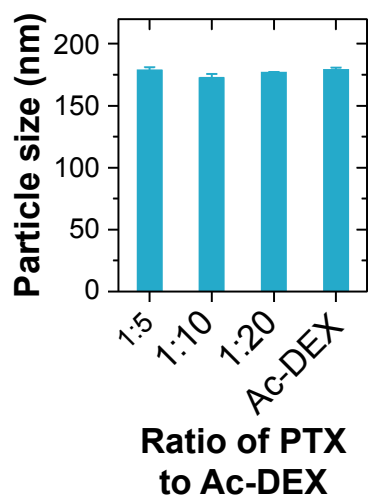

D

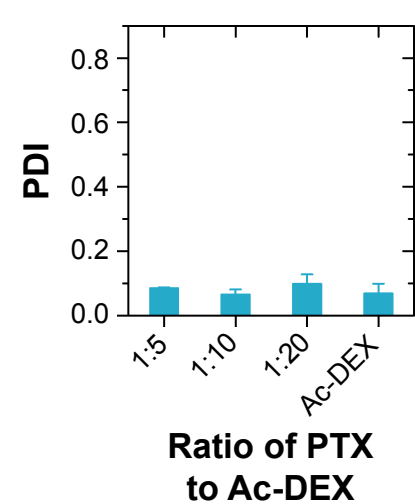

E

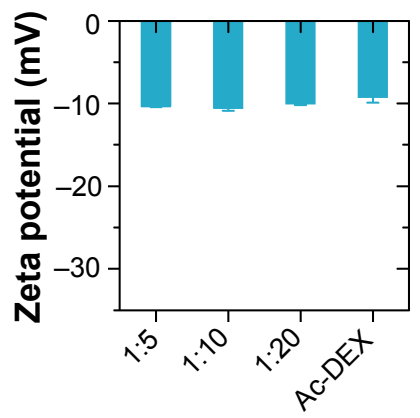

Ratio of PTX to Ac-DEX
$\mathbf{F}$

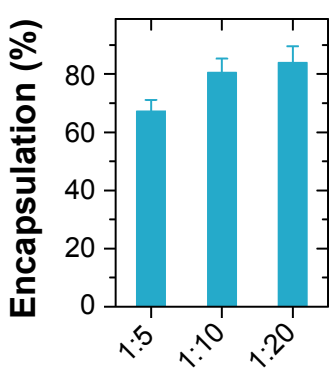

Ratio of PTX to Ac-DEX
G

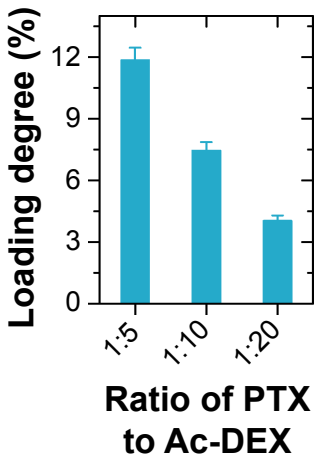

$\mathrm{H}$

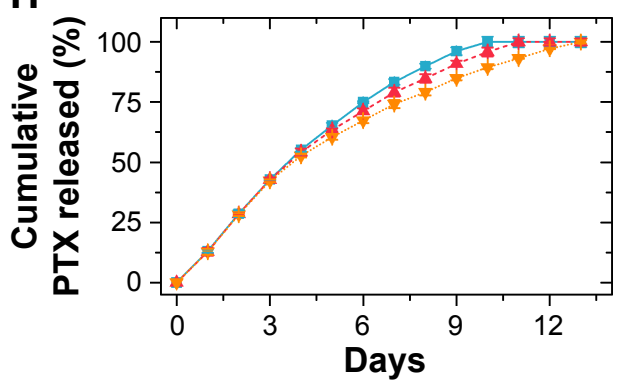

I

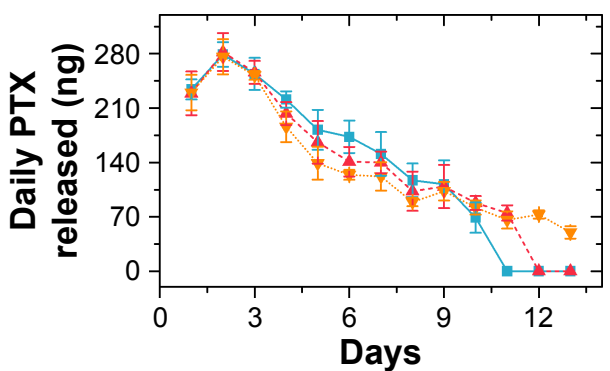

$\rightarrow-1: 5 \quad \cdots-\cdots 1: 10 \quad \cdots \cdots \cdots \cdots 1: 20$

Figure I Characterization of Ac-DEX nanoparticles and PTX release profiles.

Notes: (A) Typical transmission electron microscopy image of empty Ac-DEX nanoparticles. (B-E) The dynamic light scattering profiles (B), average particle size (C), PDI (D), and zeta-potential (E) of the Ac-DEX nanoparticles loaded with and without PTX. (F and $\mathbf{G})$ Encapsulation efficiency (F) and loading degree $(\mathbf{G})$ of PTX inside the Ac-DEX nanoparticles. ( $\mathbf{H}$ and $\mathbf{I})$ Cumulative PTX release profiles $(\mathbf{H})$ and daily PTX release profiles (I) from Ac-DEX nanoparticles in artificial cerebrospinal fluid. Data presented as mean \pm standard deviation $(n=3)$.

Abbreviations: Ac-DEX, acetalated dextran; PDI, polydispersity index; PTX, paclitaxel.

Ac-DEX. Regarding the incorporation of PTX into Ac-DEX nanoparticles,PTX@Ac-DEX, we have evaluated the impact of mass ratios between PTX and Ac-DEX (PTX:Ac-DEX) on the encapsulation efficiency and loading degree of PTX (Figure $1 \mathrm{~F}$ and $\mathrm{G}$ ). By reducing the PTX:Ac-DEX ratio from $1: 5$ to $1: 20$, the encapsulation efficiency of PTX increased from about $67 \%$ to $84 \%$, whereas the PTX loading degree decreased from about $12 \%$ to $4 \%$.

Moreover, we evaluated the effect of the PTX:Ac-DEX ratio on the release profile of PTX from PTX@Ac-DEX in aCSF (Figure 1H and I). In the first seven days, PTX@ Ac-DEX with a PTX:Ac-DEX ratio of 1:5 released approximately $87 \%$ of PTX. Similarly, approximately $79 \%$ and $74 \%$ PTX was released from PTX@Ac-DEX with PTX:Ac-DEX ratios of 1:10 and 1:20, respectively.PTX@Ac-DEX with a PTX:Ac-DEX ratio of 1:5 achieved complete release of PTX in 10 days. In contrast, PTX@Ac-DEX with a PTX:Ac-DEX ratio of 1:10 and 1:20 extended the duration of sustained release to approximately 11 and 13 days, respectively (Figure 1H). Regardless of the PTX:Ac-DEX ratio, the 
maximum release of PTX from PTX@Ac-DEX was observed on day 2 during the release test (Figure 1I). PTX@Ac-DEX with a PTX:Ac-DEX ratio of 1:5 showed the highest daily release amount from day 4 until all the PTX was released from Ac-DEX in day 10.

According to the results of encapsulation efficiency, loading degree of PTX, and PTX release profiles, we finally selected PTX@Ac-DEX with a PTX:Ac-DEX ratio of 1:5 as the formulation for the following in vitro and in vivo tests due to its most constant daily release, which is needed in vivo for sustained release for seven days.

\section{In vitro cell viability and neuroprotective effect}

To determine the concentration of Ac-DEX or PTX that induces toxicity, we evaluated the viability of neurons and astrocytes after administration of different concentrations of empty Ac-DEX nanoparticles $\left(1 \times 10^{-5}\right.$ to $10 \mathrm{mg} / \mathrm{mL}$ in Ac-DEX) or Taxol (0.1-1,000 nM in PTX). Surprisingly, our findings showed that enhanced neuronal and astrocyte viability was observed after incubation with Ac-DEX nanoparticles for 24, 48, and 72 hours (Figure 2A and B). After 24 hours of incubation, notable neuronal (but not astrocyte) viability enhancement was observed at the group with the highest concentration of Ac-DEX nanoparticles $(10 \mathrm{mg} / \mathrm{mL})$. Furthermore, when the incubation time was extended to 48 hours, a remarkable neuronal viability increased for Ac-DEX nanoparticles at a concentration of $1 \mathrm{mg} / \mathrm{mL}$ and a notable increase of astrocyte viability at high concentrations ( 1 and $10 \mathrm{mg} / \mathrm{mL}$ ) was observed. The cell viability enhancement effect for Ac-DEX nanoparticles disappeared after increasing the incubation time to 96 hours. We ascribe this disappearance of cell viability enhancement effect to the accumulation of byproducts of cellular metabolism inside the medium. The viability test suggested a neuroprotective effect of Ac-DEX nanoparticles, especially at a high concentration ( $1 \mathrm{mg} / \mathrm{mL}$ ). At the same time, we found that PTX induced immediate cytotoxicity when the concentration is above $500 \mathrm{nM}$ in both neurons and astrocytes (Figure 2C and D).

Accordingly, we prudently regulated the concentration of PTX at $10 \mathrm{nM}$ and evaluated the viability of neurons and astrocytes after incubation with empty Ac-DEX nanoparticles, Taxol, or PTX@Ac-DEX. The results indicated that no obvious cytotoxicity was observed in all the groups (Figure $3 \mathrm{~A}$ and $\mathrm{B}$ ).

After SCI, inhibitory factors, such as CSPGs, are upregulated at the site of injury and inhibit axonal extension across the site of injury. We tested whether PTX affects axonal extension of the primary neurons in the presence of CSPG (Figure 3C and D). Prior to plating, 24-well tissue culture plates were precoated with $1 \mu \mathrm{L}$ of CSPG $(10 \mu \mathrm{g} / \mathrm{mL})$, except for the PBS group that served as a blank control. The obtained neurons were examined by MAP2 under a fluorescence microscope. Compared with the PBS group, the average intensity of neurite extension was significantly $(P<0.001)$ reduced by CSPG. Empty Ac-DEX nanoparticles did not show any improvement in neurite extension compared with CSPG group. However, PTX significantly $(P<0.001)$ alleviated the effect of CSPG and promoted marked neurite extension in the Taxol group and the PTX@Ac-DEX group.

\section{In vivo drug concentration and neuroprotective effect}

We also determined the concentration of PTX in CSF, which has rarely been investigated in other studies. The results showed that the concentration of PTX in CSF was 260 ng/mL on the first day after administration of PTX@ Ac-DEX and underwent a slight decrease every day until reaching a concentration of $90 \mathrm{ng} / \mathrm{mL}$ on the seventh day after administration (Figure 4A).

To verify the neuroprotective effect of PTX, we performed a functional motor assessment and histological injury study in SD rats undergoing a weight-drop injury of the thoracic spinal cord (T10). SCI group was treated with PBS and utilized as control $(n=8)$. After traumatic SCI, motor behavior was assessed by the 21-point BBB locomotor rating scale in an open field. Complete hindlimb paralysis (BBB score $=0$ ) was observed for all four groups at days 1 and 3 postinjury. Rats in the Taxol group and the PTX@Ac-DEX group exhibited significant improvement in the BBB score compared with rats in the SCI group and Ac-DEX group from the first week postsurgery until sacrifice at the fourth week (Figure 4B).

To further understand the anatomical basis of the observed locomotor recovery, we examined the density of astrocytes, neurons, and axons that play crucial roles in spinal cord damage and repair. Neurofilaments are cell-type-specific proteins in the CNS and qualify as potential surrogate markers of damage to neurons and axons. ${ }^{30}$ The immunostaining analysis of the $200 \mathrm{kDa}$ subunit of a neurofilament (NF200), which contributes to anomalous electrophoretic mobility, has been used to evaluate neuronal and axonal damage. ${ }^{31}$ The decrease in the staining against NF200 in the lesion areas compared with the distant area as assessed by average pixel intensity values was much lower in Taxol and PTX@Ac-DEX group than PBS and Ac-DEX group $(P<0.001)$, meaning that Taxol and PTX@Ac-DEX promoted axonal regeneration after SCI 

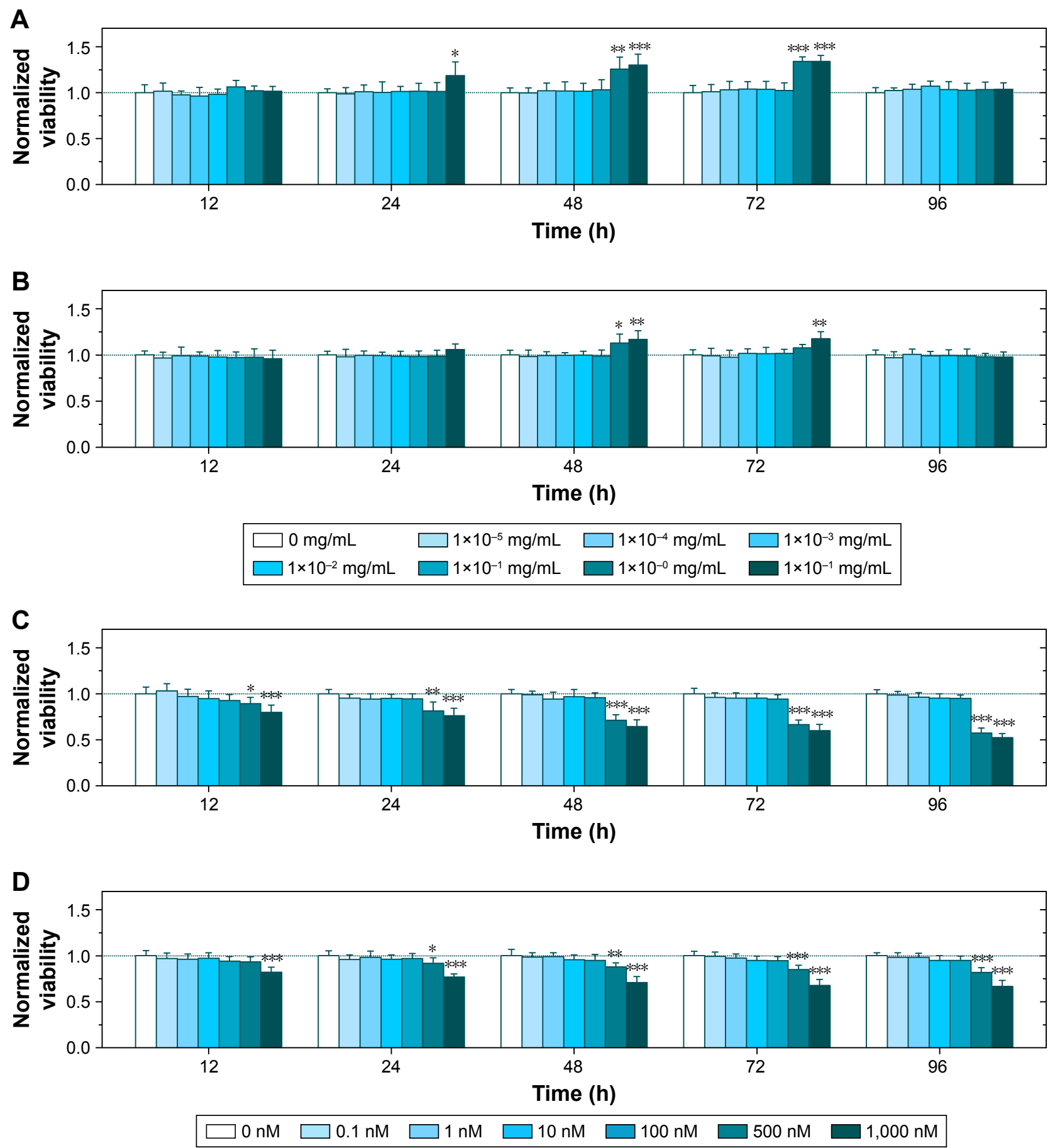

Figure 2 Ac-DEX nanoparticles protect neurons and astrocytes, and a high concentration of Taxol ${ }^{\circledR}(>500$ nM) induces immediate cytotoxicity for both cells.

Notes: (A-B) Neuronal (A) and astrocyte viability (B) under different concentrations of Ac-DEX nanoparticles. (C-D) Neuronal (C) and astrocyte viability (D) under different concentrations of Taxol. Data presented as mean \pm standard deviation $(n=6)$. $* P<0.05, * * P<0.0$ I, and $* * * P<0.00$ I.

Abbreviation: Ac-DEX, acetalated dextran.

(Figure 4C and F). Many studies showed that glial scars are impediment for axonal regeneration, and transected axons usually stop at the border of the glia-pial barrier or fibrotic scar. Therefore, functional recovery depends on axons traversing the scar barrier at the injured site. By staining CS56 to represent CSPGs, we found that CSPGs deposition was significantly reduced in the lesion area of rats in the Taxol and PTX@Ac-DEX group (Figure 4D and G). Simultaneously, we also determined the intensity of GFAP in all the groups. We found that there was no significant difference in the intensity increase of GFAP among the four groups, which indicated that the volume of the lesion area was not reduced 
A

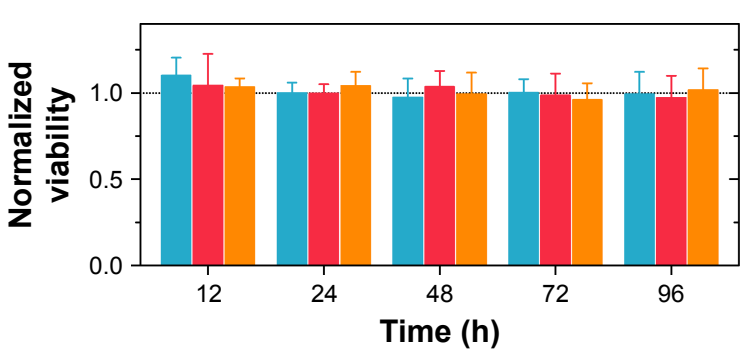

B

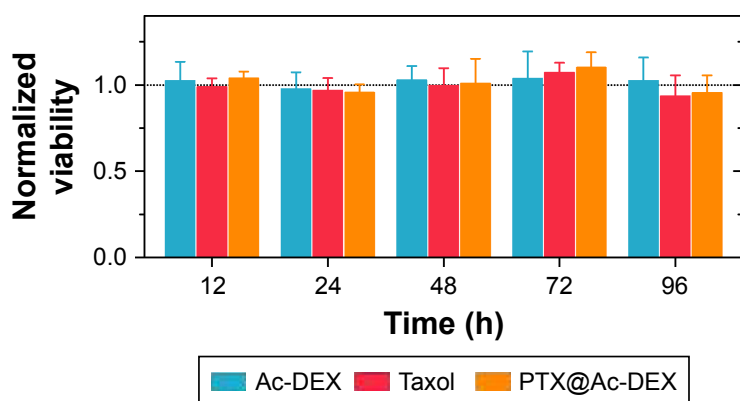

C $\stackrel{\frac{0}{\pi}}{\frac{\pi}{2}}$

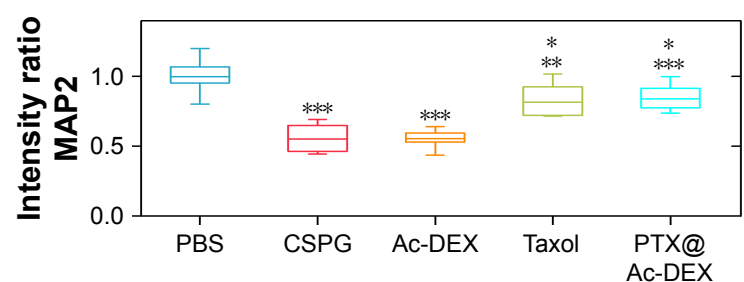

D
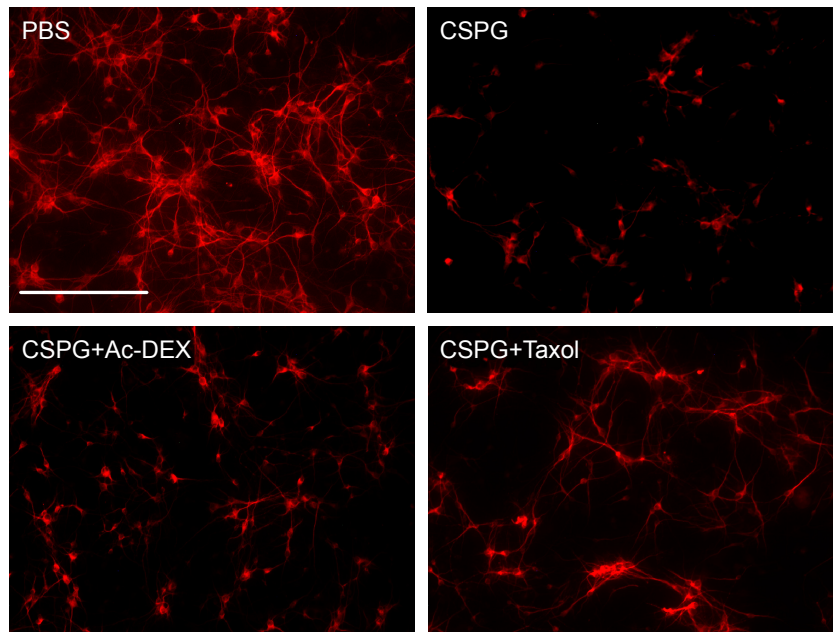

CSPG+Taxol

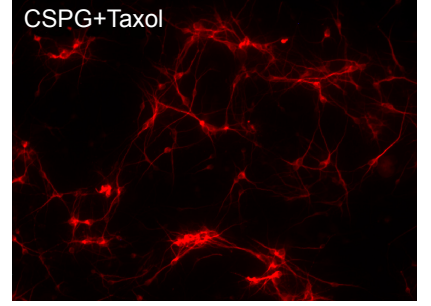

CSPG+PTX@AC-DEX

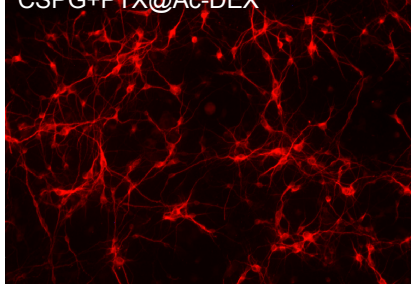

Figure 3 In vitro viability and neuroprotective effect of PTX@Ac-DEX ( $n=6)$.

Notes: (A and B) Ac-DEX nanoparticles, Taxol ${ }^{\circledR}$, and PTX@Ac-DEX did not affect the viability of neurons $(\mathbf{A})$ and astrocyte $(\mathbf{B})$ when the concentration of PTX was at 10 nM. (C) Intensity ratio of MAP2 compared with PBS group indicated an improvement of neurite extension in Taxol and PTX@Ac-DEX group. (D) Typical images of axonal regeneration in the presence of CSPG. Scale bar $=200 \mu \mathrm{m}$. Data presented as mean \pm standard deviation $(\mathrm{n}=6)$. The intensity of MAP2 incubated with CSPG and Ac-DEX was compared with the group of PBS, the intensity of MAP2 incubated with Taxol was compared with the group of CSPG, and the intensity of MAP2 incubated with PTX@ Ac-DEX was compared with the group of Ac-DEX $(* P<0.05$, **P<0.01, and $* * * P<0.001)$.

Abbreviations: Ac-DEX, acetalated dextran; CSPG, chondroitin sulfate proteoglycans; MAP2, microtubule-associated protein-2; PBS, phosphate buffer saline; PTX, paclitaxel.

by Taxol or PTX@Ac-DEX (Figure 4E-G). Therefore, PTX shows therapeutic effect by promoting axonal extension and reducing the inhibitory molecular deposition in the damaged area. Single dose of PTX@Ac-DEX administered through intrathecal injection showed equal therapeutic effect with Taxol, which need to be administered for seven days using a miniosmotic pump.

\section{Discussion}

SCI can lead to devastating disabilities, such as severe motor deficits, irreversible blindness, or paralysis., ${ }^{2,32}$ Following SCI, upregulation of extrinsic factors, including CSPGs and MAIs at the injured site of the CNS, affects the functional recovery by limiting axonal outgrowth and preventing reformation of functional synapses. ${ }^{33-35}$ CSPGs induce outgrowth inhibition through activation of Rho/ROCK signaling cascade to a depolymerization of actin filaments in growth cones and subsequent growth inhibition..$^{36-38}$ Furthermore, expression levels of CSPGs are known to typically peak in the first 2 weeks and continue 4 weeks or more after SCI. CSPGs also contribute to the formation of perineuronal nets, which can limit axonal growth and control plasticity in CNS. ${ }^{6}$ Thus, a common strategy to enhance axon regrowth is the stabilization of growth cones by preventing actin filament depolymerization by inhibitory molecules. ${ }^{33,34,36-38}$ Microtubules and their dynamic recombination are crucial for axonal outgrowth in the CNS because the stability of axonal microtubules directly determines if the impaired axons could develop regenerative growth cone favorably. ${ }^{39}$ Taxol is a Food and Drug Administration-approved anticancer drug that stabilizes microtubules and results in the interference of cell division and migration. Recent data indicated that low dose of Taxol could enhance polymerization of microtubules in growth cones without causing toxicity and thereby improving axonal growth of mature CNS neurons. ${ }^{9}$ Here, we demonstrated that CSPGs were downregulated and degraded using 

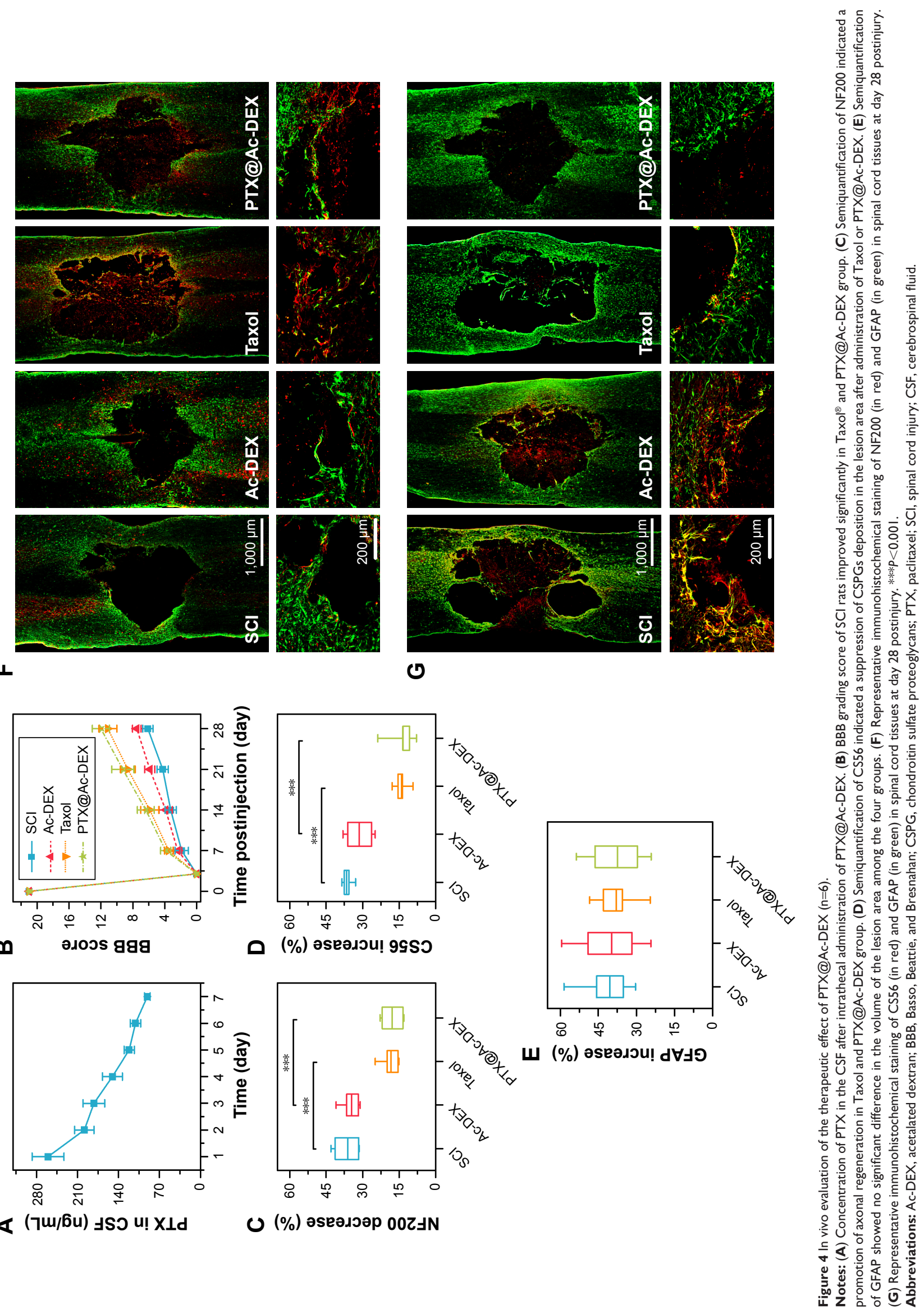

山
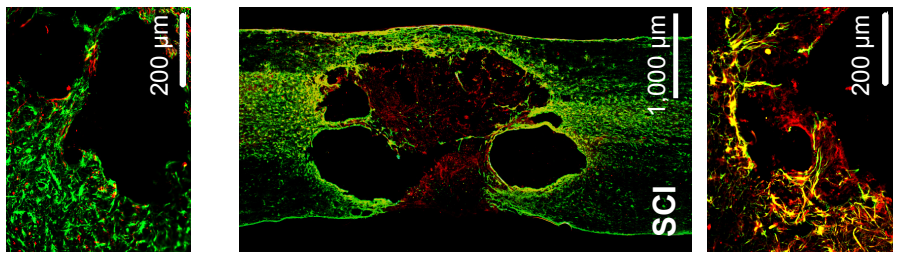

(
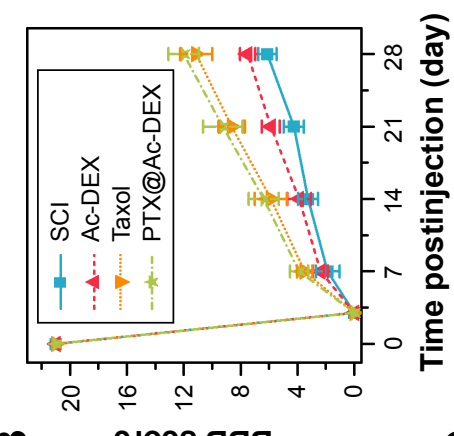

m วग0วs gag
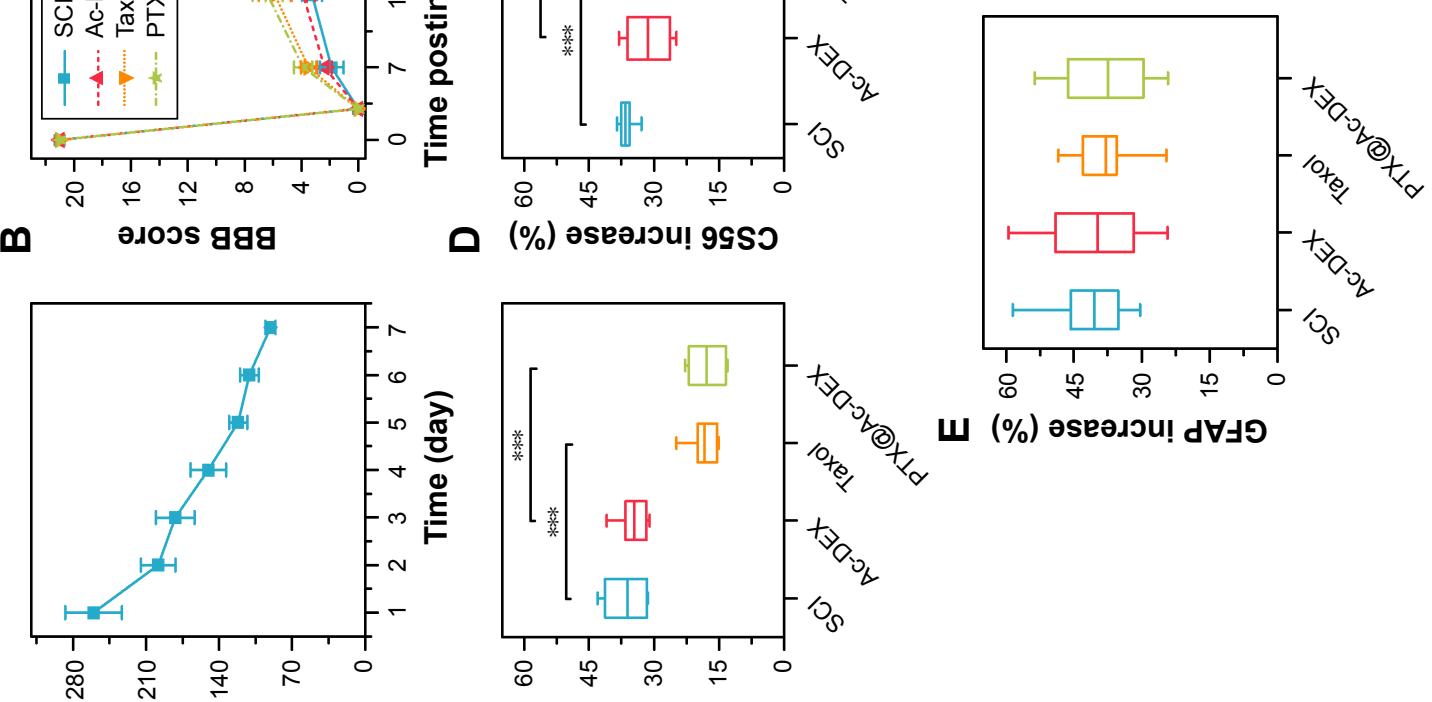

$\varangle(7 \mathrm{w} / 6 u) \exists S J \mathrm{u!} X \perp d$

৩ (\%) әsеәдәәр 00ZコN

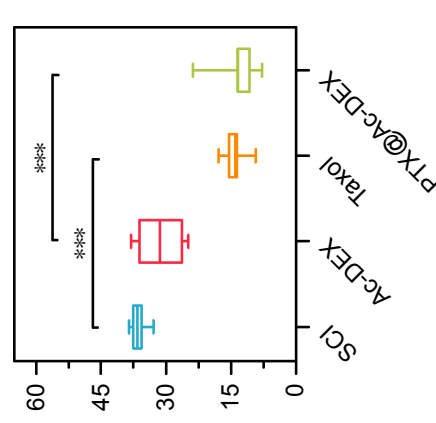


PTX@Ac-DEX nanoparticles, and as a result, PTX@AcDEX could further lead to increased axonal regeneration.

Delivery of Taxol to the injured site has been achieved using an invasive intrathecal osmatic minipump; ${ }^{9,40}$ however, this strategy accompanied with high risk of severe side effects such as compression of the spinal cord, inflammation, and increased scarring. The unique characteristics of delivering drugs to the injured sites, using biomaterials that can achieve a sustained and reliable release with only single injection, may provide an alternative and convenient therapeutic strategy compared to the conventional one. ${ }^{13,16,18,41}$ Ac-DEX nanoparticles were chosen to encapsulate PTX for the wellestablished preparation methods and the reported biomedical applications, which allow for tunable control and a longer release period in immunotherapy and myocardial infarction treatment. ${ }^{21,23,24}$ The release rate of PTX from PTX@,Ac-DEX is determined by the mass ratio between PTX and Ac-DEX. We selected PTX@Ac-DEX with a PTX:Ac-DEX ratio of $1: 5$ as the formulation in the in vitro and in vivo study because the nanoparticles presented the most constant daily release of PTX. When SCI occurs, various inhibitory factors are expressed or upregulated that prevent axonal extension such as CSPGs and MAIs. ${ }^{12}$ To properly mimic the inhibitory effects presented after an SCI, neurons were cultured in the presence of CSPGs in vitro. We demonstrated that administration of PTX@Ac-DEX could overcome the inhibitory environment and promote neurite extension.

A novel aspect of our study is that we determined the concentration of PTX in the CSF of rats in vivo during the first seven days postinjury, which is rarely done in other comparable studies. ${ }^{13,19,42}$ The result showed that the concentration of PTX in the CSF was $260 \mathrm{ng} / \mathrm{mL}$ in the first day after administration of PTX@Ac-DEX, then it underwent a slight decrease day by day until a concentration of $90 \mathrm{ng} / \mathrm{mL}$ was reached at the seventh day. The highest concentration of PTX was about $260 \mathrm{ng} / \mathrm{mL}$ on the first day, which was nontoxic according to the CCK-8 results. This relatively stable concentration may play an important role in the protection of neurons.

The immunofluorescence results demonstrated that local administration of PTX@Ac-DEX promoted axonal regeneration, which was consistent with the results of in vitro cell culture. The regeneration of axons was not observed in the Ac-DEX group when compared with our previous study, ${ }^{43}$ in which Ac-DEX alone could exhibit neuroprotective effect. This phenomenon could be ascribed to the lower concentration of Ac-DEX that has been administered in vivo than that in our previous study. Specifically, Ac-DEX nanoparticles showed a neuroprotective effect at a high concentration (above $1 \mathrm{mg} / \mathrm{mL}$ ), which was much higher than that of the in vivo study (about $30 \mu \mathrm{g} / \mathrm{L}$ ), and this evident difference in concentration contributed to the contrasting results.

Glial scar formation is one of the major impediments that hinders axon regeneration after SCI, and it is generally believed that successful regeneration of injured CNS axons can only be achieved by reducing astrogliotic scarring. . $^{33,34}$ CSPGs, major inhibitor of axonal growth, are upregulated following SCI. The signaling cascades are initiated after the binding of CSPGs to the receptors on axonal growth cones, which lead to the retraction of growth cones and formation of retraction bulb-like structures by disruption of microtubules. ${ }^{44}$ Local application of Taxol at the injury site of the optic nerve of rats enhanced axons to extend beyond the injury site and suppressed inflammation. ${ }^{45}$ Our study confirmed that PTX suppressed the expression of CSPGs in the injured spinal cord and thereby promoted axonal extension. Taxol might inhibit transforming growth factor beta signaling, which is important for the formation of inhibitory scarring. ${ }^{46}$ Similar to other studies, there was no obvious reduction of astrogliosis at 28 days postinjury in the injured spinal cord of rats treated with continuous delivery of Taxol. ${ }^{9,40}$ However, the intensity of GFAP labeling was reported to increase at the lesion site at the seventh day postinjury in a previous report, ${ }^{40}$ which was different from the 28th day in the Taxol-treated group. A recent study confirmed that astrocytes exhibit three periods including naïve astrocytes, reactive astrocytes, and scar-forming astrocytes. ${ }^{47}$ According to previous studies and our study, PTX may promote astrogliosis in the early stage only. Our study demonstrated that, by incorporation of PTX into Ac-DEX nanoparticles, low concentration of PTX promoted axonal extension and suppressed CSPGs with a single injection, which is more convenient and safer than invasive osmatic minipumps. Further studies are needed to elucidate the mechanism and leverage the multiple pathways that regulate microtubule stabilization.

\section{Conclusion}

Although preliminary studies have shown that PTX can promote neurite extension at a low concentration, we proposed a promising strategy for treating SCI with sustained release of PTX from biodegradable and neuroprotective nanoparticles. We demonstrated that administration of PTX@ Ac-DEX to the lesion site after SCI could reduce the inhibitory effect of CSPGs, enhance neural regeneration, provide neuroprotection to the injured spinal cord, and improve locomotor recovery. PTX can be released continuously for 
seven days at the injured site after single injection of PTX@ Ac-DEX nanoparticles, which showed equal therapeutic effect with continuous delivery of Taxol to the injured site for seven days using osmotic minipump. Furthermore, no Cremophor EL was involved in the formulation, which means the elimination of Cremophor-related toxicity. Overall, this study opens the perspective on the application of PTX@AcDEX for the treatment of severe neurological diseases.

\section{Acknowledgment}

We acknowledge financial support from the National Natural and Science Foundation (grant No 81371967), the "333 High Level Talents Project" in Jiangsu Province, China (grant No BRA2016512), the Jiangsu Province Six Talents Peak from Department of Human Resources, Social Security Office of Jiangsu Province, China (grant No 2014-WSN-012) and Career Development Support Program for Young and Middleaged Teachers of Shenyang Pharmaceutical University (No ZQN2015027).

\section{Disclosure}

The authors report no conflicts of interest in this work.

\section{References}

1. Fitzharris M, Cripps RA, Lee BB. Estimating the global incidence of traumatic spinal cord injury. Spinal Cord. 2014;52(2):117-122.

2. Giger RJ, Hollis ER, Tuszynski MH. Guidance molecules in axon regeneration. Cold Spring Harb Perspect Biol. 2010;2(7):a001867.

3. Schwab ME, Bartholdi D. Degeneration and regeneration of axons in the lesioned spinal cord. Physiol Rev. 1996;76(2):319-370.

4. Burda JE, Sofroniew MV. Reactive gliosis and the multicellular response to CNS damage and disease. Neuron. 2014;81(2):229-248.

5. Mothe AJ, Tator $\mathrm{CH}$. Advances in stem cell therapy for spinal cord injury. J Clin Invest. 2012;122(11):3824-3834.

6. Bradbury EJ, Moon LD, Popat RJ, et al. Chondroitinase ABC promotes functional recovery after spinal cord injury. Nature. 2002;416(6881): 636-640.

7. Shumsky JS, Tobias CA, Tumolo M, Long WD, Giszter SF, Murray M. Delayed transplantation of fibroblasts genetically modified to secrete BDNF and NT-3 into a spinal cord injury site is associated with limited recovery of function. Exp Neurol. 2003;184(1):114-130.

8. Varma AK, Das A, Wallace G, et al. Spinal cord injury: a review of current therapy, future treatments, and basic science frontiers. Neurochem Res. 2013;38(5):895-905.

9. Hellal F, Hurtado A, Ruschel J, et al. Microtubule stabilization reduces scarring and causes axon regeneration after spinal cord injury. Science. 2011;331(6019):928-931.

10. Singla AK, Garg A, Aggarwal D. Paclitaxel and its formulations. Int $J$ Pharm. 2002;235(1-2):179-192.

11. Führmann T, Anandakumaran PN, Shoichet MS. Combinatorial therapies after spinal cord injury: how can biomaterials help? Adv Healthc Mater. 2017;6(10):1601130.

12. Wilems TS, Sakiyama-Elbert SE. Sustained dual drug delivery of anti-inhibitory molecules for treatment of spinal cord injury. J Control Release. 2015;213:103-111.

13. Li H, Ham TR, Neill N. A Hydrogel bridge incorporating immobilized growth factors and neural stem/progenitor cells to treat spinal cord. $A d v$ Healthc Mater. 2016;5(7):802-812.
14. Ropper AE, Thakor DK, Han I, et al. Defining recovery neurobiology of injured spinal cord by synthetic matrix-assisted hMSC implantation. Proc Natl Acad Sci U S A. 2017;114(5):E820-E829.

15. Langer R. Drug delivery and targeting. Nature. 1998;392(6679 Suppl): $5-10$.

16. Liu D, Jiang T, Cai W, et al. An in situ gelling drug delivery system for improved recovery after spinal cord injury. Adv Healthc Mater. 2016;5(12):1513-1521.

17. Zhang S, Anderson MA, Ao Y, et al. Tunable diblock copolypeptide hydrogel depots for local delivery of hydrophobic molecules in healthy and injured central nervous system. Biomaterials. 2014;35(6): 1989-2000.

18. Kim YT, Caldwell JM, Bellamkonda RV. Nanoparticle-mediated local delivery of methylprednisolone after spinal cord injury. Biomaterials. 2009;30(13):2582-2590.

19. Roman JA, Reucroft I, Martin RA, Hurtado A, Mao HQ. Local release of paclitaxel from aligned, electrospun microfibers promotes axonal extension. Adv Healthc Mater. 2016;5(20):2628-2635.

20. Li X, Liu S, Zhao Y, et al. Training neural stem cells on functional collagen scaffolds for severe spinal cord injury repair. Adv Funct Mater. 2016;26(32):5835-5847.

21. Bachelder EM, Beaudette TT, Broaders KE, Dashe J, Fréchet JM. Acetalderivatized dextran: an acid-responsive biodegradable material for therapeutic applications. J Am Chem Soc. 2008;130(32):10494-10495.

22. Bachelder EM, Pino EN, Ainslie KM. Acetalated dextran: a tunable and acid-labile biopolymer with facile synthesis and a range of applications. Chem Rev. 2017;117(3):1915-1926.

23. Broaders KE, Cohen JA, Beaudette TT, Bachelder EM, Fréchet JM. Acetalated dextran is a chemically and biologically tunable material for particulate immunotherapy. Proc Natl Acad Sci U S A. 2009;106(14): $5497-5502$.

24. Suarez S, Grover GN, Braden RL, Christman KL, Almutairi A. Tunable protein release from acetalated dextran microparticles: a platform for delivery of protein therapeutics to the heart post-MI Biomacromolecules. 2013;14(11):3927-3935.

25. Liu D, Chen J, Jiang T, et al. Biodegradable spheres protect traumatically injured spinal cord by alleviating the glutamate-induced excitotoxicity. Adv Mater. 2018.

26. Takei N, Numakawa T, Kozaki S, et al. Brain-derived neurotrophic factor induces rapid and transient release of glutamate through the non-exocytotic pathway from cortical neurons. J Biol Chem. 1998; 273(42):27620-27624.

27. Wang YC, Wu YT, Huang HY, et al. Sustained intraspinal delivery of neurotrophic factor encapsulated in biodegradable nanoparticles following contusive spinal cord injury. Biomaterials. 2008;29(34): 4546-4553.

28. Basso DM, Beattie MS, Bresnahan JC. A sensitive and reliable locomotor rating scale for open field testing in rats. J Neurotrauma. 1995; 12(1):1-21.

29. Ceaglio N, Orozco G, Etcheverrigaray M, et al. High performance collection of cerebrospinal fluid in rats: evaluation of erythropoietin penetration after osmotic opening of the blood-brain barrier. J Neurosci Methods. 2013;219(1):70-75.

30. Petzold A. Neurofilament phosphoforms: surrogate markers for axonal injury, degeneration and loss. J Neurol Sci. 2005;233(1-2):183-198.

31. Posmantur R, Hayes RL, Dixon CE, Taft WC. Neurofilament 68 and neurofilament 200 protein levels decrease after traumatic brain injury. J Neurotrauma. 1994;11(5):533-545.

32. Maier IC, Schwab ME. Sprouting, regeneration and circuit formation in the injured spinal cord: factors and activity. Philos Trans $R$ Soc Lond B Biol Sci. 2006;361(1473):1611-1634.

33. Yiu G, He Z, Zg H. Glial inhibition of CNS axon regeneration. Nat Rev Neurosci. 2006;7(8):617-627.

34. Silver J, Miller JH. Regeneration beyond the glial scar. Nat Rev Neurosci. 2004;5(2):146-156.

35. Sharma K, Selzer ME, Li S, Sx L. Scar-mediated inhibition and CSPG receptors in the CNS. Exp Neurol. 2012;237(2):370-378. 
36. Atwal JK, Pinkston-Gosse J, Syken J, et al. PirB is a functional receptor for myelin inhibitors of axonal regeneration. Science. 2008; 322(5903):967-970.

37. Benson MD, Romero MI, Lush ME, Lu QR, Henkemeyer M, Parada LF. Ephrin-B3 is a myelin-based inhibitor of neurite outgrowth. Proc Natl Acad Sci U S A. 2005;102(30):10694-10699.

38. Dickendesher TL, Baldwin KT, Mironova YA, et al. NgR1 and NgR3 are receptors for chondroitin sulfate proteoglycans. Nat Neurosci. 2012;15(5):703-712.

39. Dent EW, Gertler FB. Cytoskeletal dynamics and transport in growth cone motility and axon guidance. Neuron. 2003;40(2):209-227.

40. Popovich PG, Tovar CA, Lemeshow S, Yin Q, Jakeman LB. Independent evaluation of the anatomical and behavioral effects of Taxol in rat models of spinal cord injury. Exp Neurol. 2014;261:97-108.

41. Liddelow SA, Guttenplan KA, Clarke LE, et al. Neurotoxic reactive astrocytes are induced by activated microglia. Nature. 2017;541(7638): $481-487$.

42. Xu B, Zhao Y, Xiao Z, et al. A dual functional scaffold tethered with EGFR antibody promotes neural stem cell retention and neuronal differentiation for spinal cord injury repair. Adv Healthc Mater. 2017;6(9):1601279.
43. Liu D, Chen J, Jiang T, et al. Biodegradable spheres protect traumatically injured spinal cord by alleviating the glutamate-induced excitotoxicity. Adv Mater. 2018;30(14):1706032.

44. Ertürk A, Hellal F, Enes J, Bradke F. Disorganized microtubules underlie the formation of retraction bulbs and the failure of axonal regeneration. J Neurosci. 2007;27(34):9169-9180.

45. Sengottuvel V, Leibinger M, Pfreimer M, Andreadaki A, Fischer D. Taxol facilitates axon regeneration in the mature CNS. J Neurosci. 2011;31(7):2688-2699.

46. Schachtrup C, Ryu JK, Helmrick MJ, et al. Fibrinogen triggers astrocyte scar formation by promoting the availability of active TGF-beta after vascular damage. J Neurosci. 2010;30(17):5843-5854.

47. Hara M, Kobayakawa K, Ohkawa Y, et al. Interaction of reactive astrocytes with type I collagen induces astrocytic scar formation through the integrin-N-cadherin pathway after spinal cord injury. Nat Med. 2017; 23(7):818-828.
International Journal of Nanomedicine

\section{Publish your work in this journal}

The International Journal of Nanomedicine is an international, peerreviewed journal focusing on the application of nanotechnology in diagnostics, therapeutics, and drug delivery systems throughout the biomedical field. This journal is indexed on PubMed Central, MedLine, CAS, SciSearch ${ }^{\circledR}$, Current Contents ${ }^{\circledR} /$ Clinical Medicine,

\section{Dovepress}

Journal Citation Reports/Science Edition, EMBase, Scopus and the Elsevier Bibliographic databases. The manuscript management system is completely online and includes a very quick and fair peer-review system, which is all easy to use. Visit http://www.dovepress.com/ testimonials.php to read real quotes from published authors. 